Internat. J. Math. \& Math. Sci.

Vol. 24, No. 10 (2000) 691-697

S0161171200003653

(C) Hindawi Publishing Corp.

\title{
LONG CYCLES IN CERTAIN GRAPHS OF LARGE DEGREE
}

\author{
PAK-KEN WONG
}

(Received 20 March 1998)

\begin{abstract}
Let $G$ be a connected graph of order $n$ and $X=\{x \in V: d(x) \geq n / 2\}$. Suppose $|X| \geq 3$ and $G$ satisfies the modified Fan's condition. We show that the vertices of the block $B$ of $G$ containing $X$ form a cycle. This generalizes a result of Fan. We also give an efficient algorithm to obtain such a cycle. The complexity of this algorithm is $O\left(n^{2}\right)$. In case $G$ is 2-connected, the condition $|X| \geq 3$ can be removed and $G$ is hamiltonian.
\end{abstract}

Keywords and phrases. Cycle, hamiltonian graph, bipartite graph, block, adjacency list, modified Fan's condition.

2000 Mathematics Subject Classification. Primary 05C38, 68R10.

1. Introduction. We consider only finite undirected graphs without loops or multiple edges. Our terminology is standard and can be found in $[4,8]$. Let $G=(V, E)$ be a graph of order $n(=|V|)$. For each vertex $x \in V$, let $N(x)=\{v \in V: v$ is adjacent to $x\}$. Then $d(x)=|N(x)|$ is the degree (valency) of $x$ in $G$. Denote by $\operatorname{dist}(x, y)$ the distance between $x$ and $y$ in $G(x, y \in V)$.

A graph $G$ is said to satisfy Fan's condition, if $\min \{\max \{d(x), d(y)\}: \operatorname{dist}(x, y)=$ $2(x, y \in V)\} \geq n / 2$. In [7], it was shown that a 2-connected graph which satisfies Fan's condition is hamiltonian. Fan's theorem is a direct generalization of Dirac's theorem [4, page 54, Theorem 4.3] and it opened an entirely new approach to study hamiltonian graphs. In [3], Fan's theorem was strengthened, where the same conditions were shown to imply the graph is pancyclic, with a few minor exceptions (also see [1]). Some generalizations of Fan's theorem can be found in [2, 5, 11, 12]. A similar result is obtained for bipartite graphs [13].

The purpose of this paper is to generalize Fan's theorem and give an algorithm to find a hamiltonian cycle. Let $X=\{x \in V: d(x) \geq n / 2\}$. Suppose $G$ is a connected graph and $|X| \geq 3$. We show that $X$ is contained in a cycle $C$ of $G$. Hence $X$ is contained in a block $B$ of $G$ (Lemma 2.3). If, in addition, $G$ satisfies the modified Fan's condition, then the vertices of $B$ form a cycle (Theorem 2.10). From this proof, we obtain an algorithm to find such a cycle. The complexity of the algorithm is $O\left(n^{2}\right)$. If $G$ is 2-connected, the condition $|X| \geq 3$ can be removed and $G$ is hamiltonian (Corollary 2.12).

2. Existence of long cycles. Let $G=(V, E)$ be a connected graph of order $n$ and $X=\{x \in V: d(x) \geq n / 2\}$.

LEMMA 2.1. If $d(u)+d(v) \geq n(u, v \in V)$ and $u$ is not adjacent to $v$, then $\mid N(u) \cap$ $N(v) \mid \geq 2$. Hence $u$ and $v$ are contained in a 4-cycle and $\operatorname{dist}(u, v)=2$. 
Proof. Suppose the lemma is not true. Then $|N(u) \cap N(v)| \leq 1$ and so

$$
n \geq d(u)+d(v)-1+|\{u, v\}| \geq n-1+2=n-1 .
$$

This is impossible and so the lemma is true.

The following lemma is probably known. For completeness, we give a proof.

LEMMA 2.2. Let $P=u_{1} u_{2} \cdots u_{k}(k \geq 3)$ be a path in $G$. If $d\left(u_{1}\right)+d\left(u_{k}\right) \geq n$, then the vertices of $P$ are contained in a cycle $C$ of $G$.

PRoof. If $u_{1}$ is adjacent to $u_{k}$, then the lemma is true. Hence we can assume that $u_{1}$ is not adjacent to $u_{k}$. If $d\left(u_{1}\right)=1$, then $d\left(u_{k}\right) \leq n-2$, which is impossible. Hence $d\left(u_{1}\right) \geq 2$ and $d\left(u_{k}\right) \geq 2$. If there exists a vertex $x \notin P$ such that $x$ is adjacent to both $u_{1}$ and $u_{k}$, then the lemma is true. Therefore, we can assume that $N\left(u_{1}\right) \cap N\left(u_{k}\right) \subset P$. By Lemma 2.1, $\left|N\left(u_{1}\right) \cap N\left(u_{k}\right)\right| \geq 2$ and so $k \geq 4$. Hence there exists some $u_{t} \in N\left(u_{1}\right)$ $\left(t \neq 2\right.$ or $k$ ) such that $u_{t-1} \in N\left(u_{k}\right)$; otherwise $d\left(u_{k}\right) \leq(n-1)-d\left(u_{1}\right)$, which is impossible. Therefore, $C=u_{1} u_{2} \cdots u_{t-1} u_{k} u_{k-1} \cdots u_{t}$ is the required cycle.

A block of a graph is a subgraph that has no cut vertices and is maximal with respect to this property (see [4, page 44]).

LEMmA 2.3. Suppose $|X| \geq 3$. Then the vertices of $X$ are contained in a cycle $C$ of $G$. Hence $X$ is contained in a block of $G$.

Proof. Let $u, v, w \in X$. If these three vertices are adjacent to each other, then we have a triangle $u v w$. If $u$ is not adjacent to $v$, then by Lemma 2.1, we have a 4-cycle containing $u$ and $v$. Hence we can assume that there is a cycle $C$ in $G$ containing at least two vertices $u, v \in X$. Suppose there exists a vertex $w \in X$ and $w \notin C$. We show that there exists a cycle $C^{\prime}$ containing the vertices $X \cap C$ and $\{w\}$. First we claim that there is a path $P$ of length at most 4 passing through $w$ and connecting two vertices of $C$ that is internally disjoint from $C$.

CASE 1. Assume $w$ is adjacent to $u$. If $w$ is also adjacent to $v$, then our claim is clearly true. Hence we can assume that $w$ is not adjacent to $v$. Then by Lemma 2.1, there is a 4-cycle containing $v$ and $w$. Therefore, there exists a vertex $x$ such that $x$ is adjacent to $v$ and $w$. If $x \in C$, then $P=x w u$; otherwise $P=v x w u$.

CASE 2. Assume that $w$ is not adjacent to $u$ or $v$. Then by Lemma 2.1, $\mid N(w) \cap$ $N(u) \mid \geq 2$ and $|N(w) \cap N(v)| \geq 2$. Hence there exist two vertices $x$ and $y$ such that $x$ is adjacent to $w$ and $u$, and $y$ is adjacent to $w$ and $v$. If both $x$ and $y \notin C$, then $P=u x w y v$; otherwise we have a shorter path. This proves our claim.

Let $\left\{w_{i}, w_{j}\right\}=P \cap C$. If the section of cycle $C$ from $w_{i}$ to $w_{j}$ contains all vertices of $X \cap C$, then $C^{\prime}=w_{i} \cdots w_{j} \cup P$ is the required cycle. Hence we can assume that the section of $C$ from $w_{i}$ to $w_{j}$ contains a vertex $u \in X \cap C$ and the section of $C$ from $w_{j}$ to $w_{i}$ contains a vertex $v \in X \cap C$. Furthermore, we can assume that the section of $C$ from $u$ to $w_{j}$ on $C$ contains no interior vertex which is in $X$ and the section of $C$ from $v$ to $w_{i}$ contains no interior vertex which is in $X$. Since $u, v \in X$, it follows from Lemma 2.2 that the path $P^{\prime}=u \cdots w_{i} \cdots w \cdots w_{j} \cdots v$ is contained in cycle $C^{\prime}$ of $G$. Since $X$ is finite, we can always obtain a cycle containing all vertices of $X$. This completes the proof. 


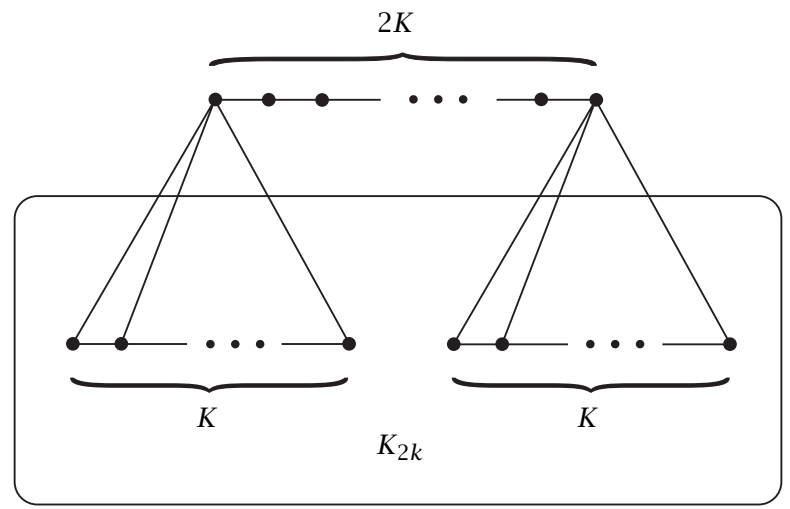

FIGURE 2.1.

REMARK 2.4. If $|X| \leq 2$, Lemma 2.3 is not true. Let $G$ be the graph with $V=\left\{v_{1}, v_{2}\right.$, $\left.v_{3}, v_{4}, v_{5}, v_{6}\right\}$ such that $v_{1}$ is adjacent to $v_{2}, v_{3}$, and $v_{4} ; v_{2}$ is adjacent to $v_{3} ; v_{4}$ is adjacent to $v_{5}$ and $v_{6} ; v_{5}$ is adjacent to $v_{6}$. Then $|X|=2$ and there is no cycle containing $X$ in $G$.

REMARK 2.5. Suppose $G$ is 2-connected. Then every two vertices of $G$ is contained in a cycle. Hence we can always find a cycle containing all vertices of $X$ by the proof of Lemma 2.3. Therefore, the condition $|X| \geq 3$ in Lemma 2.3 can be removed, if $G$ is assumed to be 2 -connected.

A graph $G$ is said to satisfy the modified Fan's condition, if for any vertex $w \in V$ with $d(w) \geq 3$, we have $x, y \in N(w)$ implies either $x$ is adjacent to $y$ or $\max \{d(x), d(y)\} \geq$ $n / 2$.

REMARK 2.6. Let $G$ be the graph with $V=\left\{v_{1}, v_{2}, v_{3}, v_{3}, v_{4}, v_{5}, v_{6}\right\}$ such that $v_{1}$ is adjacent to $v_{3}, v_{4}, v_{5}, v_{6} ; v_{2}$ is adjacent to $v_{3}, v_{4}, v_{5}, v_{6} ; v_{3}$ is adjacent to $v_{4}$. Then $|X| \geq 3$, but $G$ is not hamiltonian and $G$ does not satisfy the modified Fan's condition.

REMARK 2.7. If $G$ satisfies Fan's condition, then $G$ satisfies the modified Fan's condition, but not vise versa.

EXAMPLE 2.8. Let $n=4 k(k \geq 2)$ and $G$ the graph given in Figure 2.1. Then $G$ satisfies the modified Fan's condition, but not the Fan's condition. It is easy to see that the diameter of $G$ is equal to $k+1$. However, the diameter of any graph satisfying Fan's condition is less than or equal to 6 .

LEMmA 2.9. Suppose $|X| \geq 3$ and $G$ satisfies the modified Fan's condition. Let $C$ be a cycle containing $X$. If $z \notin C$ and $|N(z) \cap C| \geq 2$, then there exists a cycle $C^{\prime}$ containing $z$ and $C$.

Proof. Write $C=w_{1} w_{2} \cdots w_{k}(k \geq 3)$. Since $z \notin C, d(z)<n / 2$. Let $w_{i}$ and $w_{j}(i<j)$ be two vertices of $C$ which are adjacent to $z$. If $z$ is adjacent to $w_{i+1}$, then $C^{\prime}=w_{i} \cdots w_{1} \cdots w_{i+1} z$ is the required cycle. Therefore, we can assume that $z$ is not 
adjacent to $w_{i+1}, w_{i-1}, w_{j+1}$, or $w_{j-1}$. Since $d\left(w_{i}\right) \geq 3$ and $z$ is not adjacent to $w_{i+1}$, we have $d\left(w_{i+1}\right) \geq n / 2$. Similarly, $d\left(w_{j+1}\right) \geq n / 2$. Let $P=w_{i+1} \cdots w j z w_{i} w_{i-1} \cdots w_{j+1}$. Then by Lemma 2.2, there exists a cycle $C^{\prime}$ containing $P$. This proves Lemma 2.9.

THEOREM 2.10. Suppose $|X| \geq 3$ and $G$ satisfies the modified Fan's condition. Let $B$ be the block of $G$ containing $X$. Then the vertices of $B$ form a cycle.

Proof. By Lemma 2.3, there exists a cycle $C$ containing the vertices of $X$. Let $A=$ $V(B)-V(C)$ and suppose $A \neq \varnothing$. We show that there is a larger cycle $C^{\prime}$ containing all the vertices of $C$. Let $a=\max \{d(v): v \in A\}$ and write $C=w_{1} w_{2} \cdots w_{k}(k \geq 3)$.

CASE 1. Suppose $a=2$. Hence $d(v)=2$ for each $v \in A$. Let $z \in A$. Then by constructing a tree rooted at $z$, we can find a path $Q=u_{1} u_{2} \cdots u_{t}$ with $u_{1}=w_{i}, u_{t}=w_{j}$ and $u_{l} \in A(1<l<t)$. If $j=i-1$ or $i+1$, then $C^{\prime}=w_{j} \cdots w_{i} u_{2} \cdots u_{t-1}$. Hence we can assume that $w_{i}$ and $w_{j}$ are nonconsecutive vertices of $C$. Since $d\left(u_{2}\right)=2, u_{2}$ is not adjacent to $w_{i+1}$. Since $d\left(w_{i}\right) \geq 3$ and $d\left(u_{2}\right)<n / 2$, it follows that $d\left(w_{i+1}\right) \geq n / 2$. Similarly, $d\left(w_{j+1}\right) \geq n / 2$. Let $P=w_{i+1} \cdots w_{j} u_{t-1} \cdots u_{2} w_{i} \cdots w_{j+1}$. Then by Lemma 2.2, $P$ is contained in a cycle $C^{\prime}$ of $B$.

CASE 2. Suppose $a \geq 3$ and choose $z \in A$ such that $d(z)=a \geq 3$. By Lemma 2.9, we can assume $|N(z) \cap C| \leq 1$. We show that $N(z) \cap C \neq \varnothing$. Suppose this is not true. Then $d(u)<n / 2$ for all $u \in N(z)$ and so $N(z) \cup\{z\}$ forms a complete subgraph of $G$. Hence $d(u) \geq d(z)$ and by the maximality of $d(z)$, we have $d(u)=d(z)$ for all $u \in N(z)$. But $G$ is connected and this is impossible. Hence $|N(z) \cap C|=1$.

Let $\left\{w_{i}\right\}=N(z) \cap C$. Then the set $H=\left(N(z)-\left\{w_{i}\right\}\right) \cup\{z\}$ forms a complete subgraph of $G$. Hence $d(u) \geq d(z)-1$ for all $u \in H$ and $d(u)=d(z)-1$ if and only if $u$ is adjacent only to vertices of $H$. Since $w_{i}$ is not a cut vertex of $B$, there exists some vertex $v \in H$ such that $v \notin N\left(w_{i}\right)$ and $d(v)>d(z)-1$. By the maximality of $d(z)$, we have $d(v)=d(z)$. Then there exists a $y \notin H \cup\left\{w_{i}\right\}$ such that $y$ is adjacent to $v$. Suppose $d(y)<n / 2$. Since $d(z)<n / 2$ and $d(v)=d(z) \geq 3$, it follows that $y$ is adjacent to $z$. Hence $y \in H$, which is impossible. Therefore $d(y) \geq n / 2$ and so $y \in C$. Write $y=w_{j}$. Since $d(v)=d(z), v$ is not adjacent to $w_{j-1}$ or $w_{j+1}$. Let $Q=w_{i} z v w_{j}$. Then by the proof of Case 1 , we can find a larger cycle $C^{\prime}$ containing all vertices of $C$.

Since $B$ is finite, we can always obtain a cycle containing all vertices of $B$. This completes the proof of the theorem.

REMARK 2.11. If $|X| \leq 2$, then Theorem 2.10 is not true. See the example given in Remark 2.4.

By using Remark 2.5 and the proof of Theorem 2.10, we have the following result which generalizes Fan's theorem.

COROLlary 2.12. Suppose $G$ is 2-connected and satisfies the modified Fan's condition, then $G$ is hamiltonian.

3. An algorithm. In this section, let $G$ be represented by an adjacent list (see [6, page 173] or [10, page 17]) and let $P=u_{1} u_{2} \cdots u_{k}(k \geq 3)$ be a path in $G$.

ALGorithm 3.1. (If $d\left(u_{1}\right)+d\left(u_{k}\right) \geq n$, we find a cycle $C$ containing all the vertices of $P$.) 
STEP 1. Let $f(v) \neq 0$ for each $v \in N\left(u_{1}\right), g(v) \neq 0$ for each $v \in N\left(u_{k}\right)$ and $h\left(u_{i}\right) \neq 0(i=1,2, \ldots, k)$.

STEP 2. If $g\left(u_{1}\right) \neq 0$, then $u_{1} \in N\left(u_{k}\right)$ and stop.

STEP 3. If there exists a vertex $x \in N\left(u_{1}\right)$ such that $g(x) \neq 0$ and $h(x)=0$, then $x \in N\left(u_{1}\right) \cap N\left(u_{k}\right)$ and $x \notin P$. Let $C \leftarrow u_{1} u_{2} \cdots u_{k} x$ and stop.

STEP 4. Find $t(t \neq 2$ or $k)$ such that $f\left(u_{t}\right) \neq 0$ and $g\left(u_{t-1}\right) \neq 0$. Let $C \leftarrow u_{1} u_{2} \cdots$ $u_{t-1} u_{k} u_{k-1} \cdots u_{t}$ and stop.

The correctness of the algorithm follows from Lemma 2.2 and its complexity is clearly $O(n)$.

Algorithm 3.2. (Assume $|X| \geq 3$. Then the vertices of $X$ is contained in a cycle $C$ of $G$.)

STEP 1. Find a cycle $C$ containing at least two vertices in $X$.

STEP 2. Let $X^{\prime}=X-C$. If $X^{\prime}=\varnothing$, stop. Otherwise let $w \in X^{\prime}$ and $u, v \in X$.

STEP 3. Find a path $P$ of length at most 4 passing through $w$ and connecting two vertices $\left\{w_{i}, w_{j}\right\}$ of $C$ that is internally disjoint from $C$.

STEP 4. Let $Q_{1} \leftarrow w_{i} \cdots w_{j}$ and $Q_{2} \leftarrow w_{j} \cdots w_{i}$. If $Q_{2} \cap X=\varnothing$, let $C \leftarrow Q_{1} \cup P$ and go to Step 2. If $Q_{1} \cap X=\varnothing$, let $C \leftarrow Q_{2} \cup P$ and go to Step 2 .

STEP 5. Choose $u \in Q_{1}$ and $v \in Q_{2}$ such that the sections $u \cdots w_{j}$ and $v \cdots w_{i}$ of $C$ contains no interior vertex in $X$. Let $P \leftarrow u \cdots w_{i} \cdots w \cdots w_{j} \cdots v$.

STEP 6. Apply Algorithm 3.1 to the path $P$ and obtain a cycle $C$ containing all vertices of $P$. Go to Step 2.

The correctness of the algorithm follows from Lemma 2.3.

We show that Algorithm 3.2 can be implemented in $O\left(n^{2}\right)$ time. Pick $u, v, w \in X$ and set $f(x) \neq 0$ for all $x \in N(u)$ and $g(x) \neq 0$ for all $x \in N(v)$. If $f(v) \neq 0, f(w) \neq 0$ and $g(w) \neq 0$, then let $C \leftarrow u v w$. Otherwise, we can assume $v$ is not adjacent to $u$, that is, $f(v)=0$. Then by Lemma 2.1, there exist $x, y \in N(u)$ such that $g(x) \neq 0$ and $g(y) \neq 0$. Let $C \leftarrow u x v y$. Hence Step 1 takes $O(n)$ time.

Let $f(z) \neq 0$ for all $z \in N(u), g(z) \neq 0$ for all $z \in N(v), h(z) \neq 0$ for all $z \in N(w)$, and $H(z) \neq 0$ for all $z \in C$. Suppose first that $u$ is adjacent to $w$; that is, $h(u) \neq 0$. If $h(v) \neq 0$, then let $P \leftarrow u v w$. If $h(v)=0$, then by Lemma 2.1, we can find a vertex $x \in N(w)$ such that $g(x) \neq 0$. If $H(x) \neq 0$, then let $P \leftarrow x w u$; otherwise let $P \leftarrow v x w u$. Now assume that $u$ and $v$ are not adjacent to $w$, that is, $h(u)=0$ and $h(v)=0$. Then there exists two vertices $x$ and $y$ such that $x \in N(u)$ and $h(x) \neq 0, y \in N(v)$ and $h(y) \neq 0$. If both $H(x)=0$ and $H(y)=0$, let $P \leftarrow u x w y v$. Otherwise we have a shorter path $P$. Hence Step 3 takes $O(n)$ time.

It is easy to see that Steps 4,5 , and 6 can be implemented in $O(n)$ time. Combine this with Step 2, we have an $O\left(n^{2}\right)$ algorithm.

Let $B$ be the block of $G$ containing $X$. Then $B$ can be found using the depth-first search for blocks algorithm by Hopcroft and Tarjan (see [6] or [10]). The complexity of this algorithm is $O(\max (n,|E|))$. For our graphs, $|E|=O\left(n^{2}\right)$. Therefore it takes $O\left(n^{2}\right)$ time to find the block $B$ of $G$.

Algorithm 3.3. (Assume $|X| \geq 3$ and $G$ satisfies the modified Fan's condition. We find a cycle containing the vertices of $B$.) 
STEP 1. Find a cycle $C$ containing the vertices of $X$ (use Algorithm 3.2).

STEP 2. Let $A \leftarrow V(B)-V(C)$. If $A=\varnothing$, stop.

STEP 3. Let $a \leftarrow \max \{d(v): v \in A\}$ and $z \in A$ with $d(z)=a$. Write $C=w_{1} w_{2} \cdots w_{k}$ $(k \geq 3)$. If $a \geq 3$, go to Step 7 .

$\operatorname{STEP} 4(a=2)$. Find a path $Q=u_{1} u_{2} \cdots u_{t}$ such that $u_{1}=w_{i}, u_{t}=w_{j}$, and $u_{l} \in$ $A(1<l<t)$.

STEP 5. If $j=i-1$ or $i+1$, let $C \leftarrow w_{j} \cdots w_{i} u_{2} \cdots u_{t-1}$ and go to Step 2 .

STEP 6. Assume $u_{i}$ and $u_{j}$ are nonconsecutive vertices of $C$. Let $P \leftarrow w_{i+1} \cdots w_{j}$ $u_{t-1} \cdots u_{2} w_{i} \cdots w_{j+1}$. Use Algorithm 3.1 to find a cycle $C$ containing all vertices of $P$ and go to Step 2.

STEP $7(a \geq 3)$. If $|N(z) \cap C|=1$, go to Step 10 .

STEP $8(|N(z) \cap C| \geq 2)$. Let $w_{i}$ and $w_{j}$ be two vertices of $C$ which are adjacent to $z$. If $z \in N\left(w_{i+1}\right)$, then let $C \leftarrow w_{i} \cdots w_{1} \cdots w_{i+1} z$ and go to Step 2 .

STEP 9 (assume $z$ is not adjacent to $w_{i+1}, w_{i-1}, w_{j+1}$ or $w_{j-1}$ ). Let $P \leftarrow w_{i+1} \cdots w_{j}$ $z w_{i} w_{i-1} \cdots w_{j+1}$. Apply Algorithm 3.1 to find a cycle $C$ containing the vertices of $P$ and go to Step 2.

STEP 10. Let $\left\{w_{i}\right\}=N(z) \cap C$ and $H \leftarrow\left(N(z)-\left\{w_{i}\right\}\right) \cup\{z\}$. Find a vertex $v \in H$ such that $v \notin N\left(w_{i}\right)$ and $d(v)=d(z)$.

STEP 11. Find $w_{j} \in N(v)$ and $w_{j} \notin H \cup\left\{w_{i}\right\}$. Let $u_{2} \leftarrow z, u_{3} \leftarrow v, t \leftarrow 4$ and $Q \leftarrow w_{i} u_{2} u_{3} w_{j}$. Go to Step 5 .

The correctness of the algorithm follows from Theorem 2.10.

We show that the algorithm can be implemented in $O\left(n^{2}\right)$ time. Step 1 uses Algorithm 3.2 and so it takes $O\left(n^{2}\right)$ time. Step 3 can be done in $O(n)$ time. (If we use F-heaps data structure [9], it takes $O(\log n)$ time.) In Step 4, we construct a tree rooted at $z$. As soon as we find two vertices $w_{i}$ and $w_{j} \in C$, we stop. Since $a=2$, each vertex of $Q$ has degree 2. Hence $Q$ can be constructed in $O(n)$ time. It is clear that Steps 5 , $6,7,8,9,10$, and 11 can be done in $O(n)$ time. Combine this with Step 2, we have an $O\left(n^{2}\right)$ algorithm.

\section{REFERENCES}

[1] D. Bauer and E. Schmeichel, Hamiltonian degree conditions which imply a graph is pancyclic, J. Combin. Theory Ser. B 48 (1990), no. 1, 111-116. MR 91m:05126. Zbl 698.05047.

[2] P. Bedrossian, G. Chen, and R. H. Schelp, A generalization of Fan's condition for Hamiltonicity, pancyclicity, and Hamiltonian connectedness, Discrete Math. 115 (1993), no. 1-3, 39-50. MR 94e:05173. Zbl 773.05075.

[3] A. Benhocine and A. P. Wojda, The Geng-Hua Fan conditions for pancyclic or Hamilton-connected graphs, J. Combin. Theory Ser. B 42 (1987), no. 2, 167-180. MR 88f:05071. Zbl 613.05038.

[4] J. A. Bondy and U. S. R. Murty, Graph Theory with Applications, American Elsevier Publishing Co., Inc., New York, 1976. MR 54\#117.

[5] G. Chen and R. H. Schelp, Hamiltonian graphs involving distances, J. Graph Theory 16 (1992), no. 2, 121-129. MR 93b:05102. Zbl 766.05053.

[6] S. Even, Graph Algorithms, Computer Science Press, Inc., Woodland Hills, California, 1979, Computer Software Engineering Series. MR 82e:68066. Zbl 441.68072.

[7] G. H. Fan, New sufficient conditions for cycles in graphs, J. Combin. Theory Ser. B 37 (1984), no. 3, 221-227. MR 86c:05083. Zbl 551.05048. 
[8] L. R. Foulds, Graph Theory Applications, Universitext, Springer-Verlag, New York, 1992. MR 96a:05041. Zbl 747.05001.

[9] M. L. Fredman and R. E. Tarjan, Fibonacci heaps and their uses in improved network optimization algorithms, J. Assoc. Comput. Mach. 34 (1987), no. 3, 596-615. MR 90d:68012.

[10] A. Gibbons, Algorithmic Graph Theory, Cambridge University Press, Cambridge, New York, 1985. MR 87d:68090. Zbl 568.05001.

[11] R. J. Gould, Updating the Hamiltonian problem-a survey, J. Graph Theory 15 (1991), no. 2, 121-157. MR 92m:05128. Zbl 746.05039.

[12] H. J. Veldman, Short proofs of some Fan-type results, Ars Combin. 29 (1990), 28-32. MR 91g:05091. Zbl 715.05047.

[13] P.-K. Wong, Longest cycles in certain bipartite graphs, Int. J. Math. Math. Sci. 21 (1998), no. 1, 103-106. MR 98g:05083. Zbl 890.05040.

PAK-Ken Wong: DePartment of MATHematics AND COMPUTER SCIENCE, SetOn Hall UniVERSITY, SOUTH ORANGE, NJ 07079, USA 


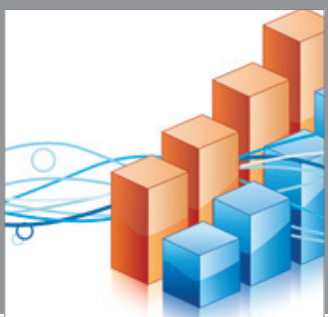

Advances in

Operations Research

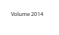

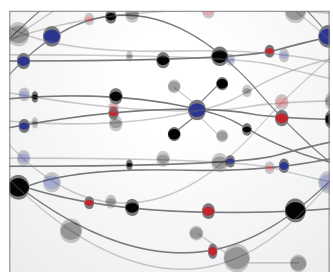

\section{The Scientific} World Journal
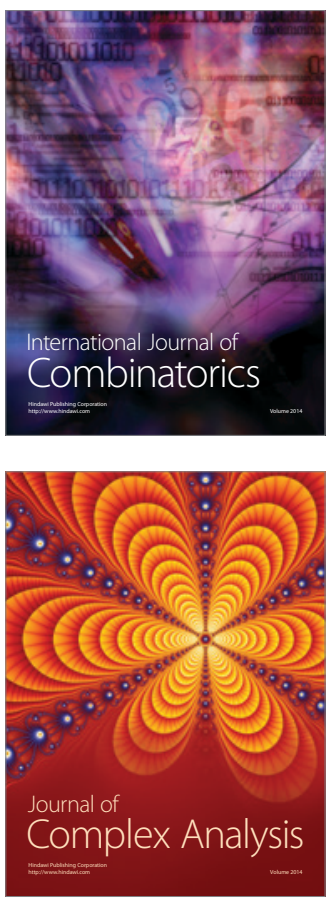

International Journal of

Mathematics and

Mathematical

Sciences
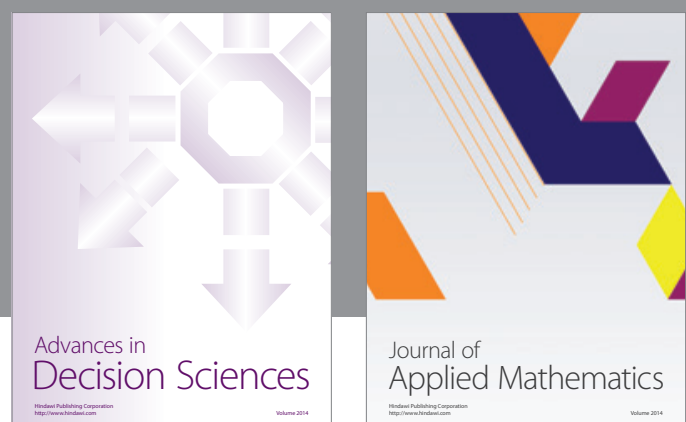

Journal of

Applied Mathematics
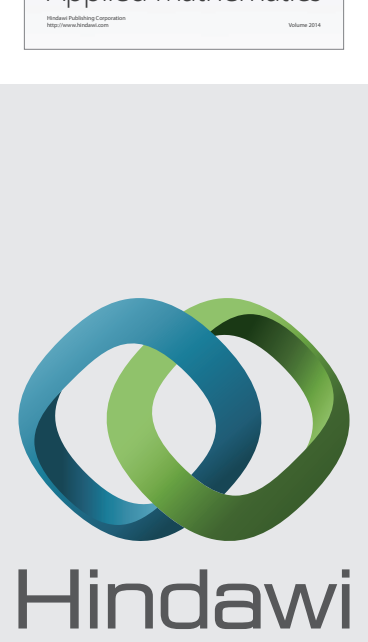

Submit your manuscripts at http://www.hindawi.com
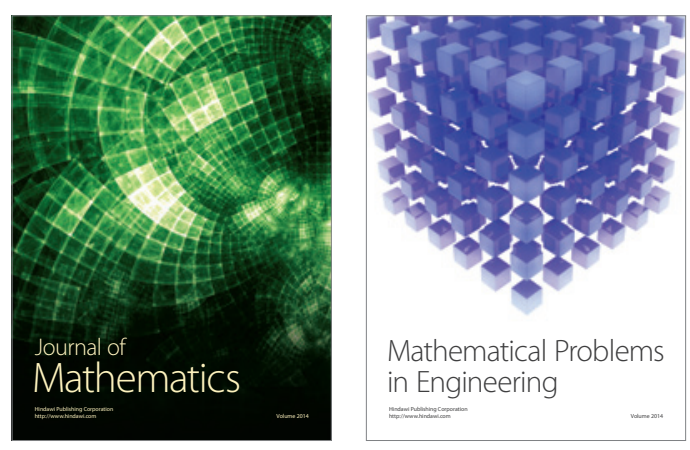

Mathematical Problems in Engineering
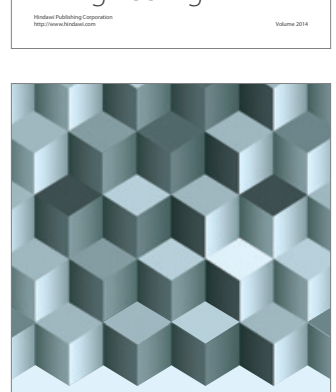

Journal of

Function Spaces
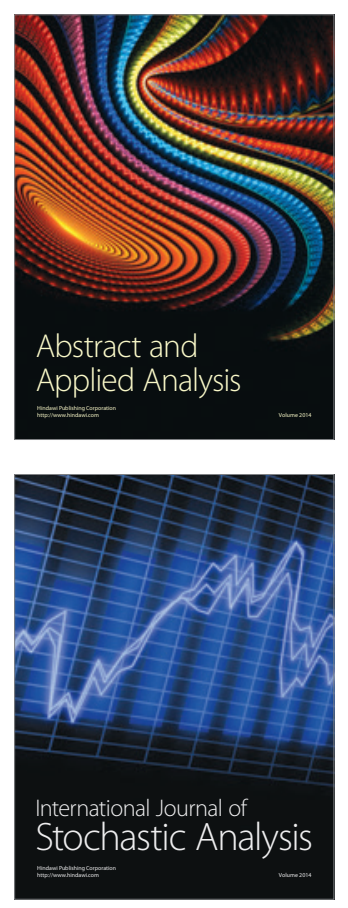

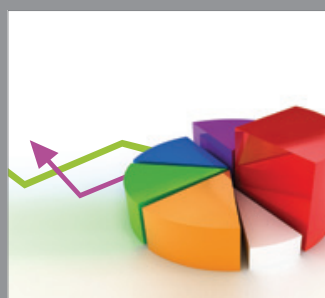

ournal of

Probability and Statistics

Promensencen
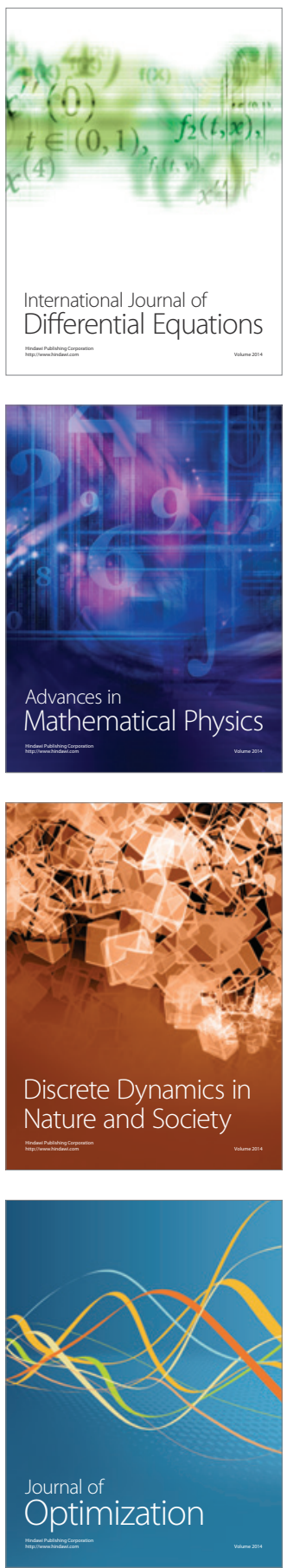